\title{
ALGEBRAIC CHARACTERIZATION OF ISOMETRIES OF THE COMPLEX AND THE QUATERNIONIC HYPERBOLIC 3-SPACES
}

\author{
KRISHNENDU GONGOPADHYAY
}

(Communicated by Michael Wolf)

\begin{abstract}
Let $\mathbf{H}_{\mathbb{F}}^{3}$ denote the three dimensional hyperbolic space over $\mathbb{F}$, where $\mathbb{F}$ denotes either the complex numbers $\mathbb{C}$ or the quaternions $\mathbb{H}$. We offer an algebraic characterization of isometries of $\mathbf{H}_{\mathbb{F}}^{3}$.
\end{abstract}

\section{INTRODUCTION}

For a group $G$, let $P G$ denote the quotient by its center, i.e. $P G=G / Z(G)$. Let $\mathbb{F}$ denote the complex numbers $\mathbb{C}$ or the quaternions $\mathbb{H}$. Let $\mathbf{H}_{\mathbb{F}}^{\mathrm{n}}$ denote the $n$-dimensional hyperbolic space over $\mathbb{F}$. For $\mathbb{F}=\mathbb{C}$ and $\mathbb{H}$, the linear groups which act as the isometries of $\mathbf{H}_{\mathbb{F}}^{\mathbf{n}}$ are denoted by $U(n, 1)$ and $S p(n, 1)$ respectively. The corresponding isometry groups are identified with $P U(n, 1)$ and $P S p(n, 1)$ respectively.

Classically, the dynamical types of isometries of the two and three dimensional real hyperbolic spaces were classified as elliptic, parabolic and hyperbolic according to the dynamics of their fixed points. This trichotomy of the isometries can be characterized algebraically in terms of their traces; cf. [Be, Theorems 4.3.1 and 4.3.4]. It is a natural question to ask for similar characterizations in the complex and the quaternionic hyperbolic geometries. An algebraic characterization of isometries of $\mathbf{H}_{\mathbb{C}}^{2}$ is now well-known due to the work of Giraud and Goldman Gol, Theorem 6.2.4]. Recently, Cao and Gongopadhyay CaGo have offered a counterpart of Goldman's theorem in the quaternionic setting. However, it is interesting to obtain a similar characterization for the isometries of $\mathbf{H}_{\mathbb{F}}^{3}$. In the complex case, such a characterization may be useful to calculate the limit sets of the 4-dimensional complex Kleinian groups; cf. [SV]. In this context, Goldman's characterization has been generalized to $S L(3, \mathbb{C})$ action on $\mathbb{P}^{3}$ in $[\mathrm{Na}$. In order to generalize this classification in dimension 4 , the starting point is to look for counterparts of Goldman's theorem in three dimensional complex hyperbolic geometry. In this paper, we offer an algebraic characterization of the isometries of $\mathbf{H}_{\mathbb{F}}^{\mathbf{3}}$. Along the way we obtain a simple criterion to determine the fixed-point trichotomy of the isometries in all dimensions.

Received by the editors March 31, 2011 and, in revised form, July 24, 2011.

2010 Mathematics Subject Classification. Primary 51M10; Secondary 15B57, 53C35.

Key words and phrases. Isometry, complex and quaternionic hyperbolic space, classification.

The author gratefully acknowledges the support of SERC-DST FAST grant SR/FTP/MS$004 / 2010$. 
TABLE 1. Classification of elliptic isometries of $\mathbf{H}_{\mathbb{H}}^{3}$

\begin{tabular}{|l|c|}
\hline Classification & Nature of eigenvalues \\
\hline Regular elliptic & mutually distinct (classes of) eigenvalues \\
\hline Compound elliptic & two and only two equal eigenvalues \\
\hline Complex elliptic & a pair of equal eigenvalues \\
\hline Screw elliptic & has an eigenvalue of multiplicity three \\
\hline Simple elliptic & has a single class of eigenvalues \\
\hline
\end{tabular}

When $\mathbb{F}=\mathbb{H}$, the underlying Hermitian space $\mathbb{H}^{2,1}$ is always assumed to be a right vector space over $\mathbb{H}$. Accordingly, the eigenvalues of $g$ in $S p(3,1)$ are assumed to be right eigenvalues.

Let $g$ be an isometry of $\mathbf{H}_{\mathbb{F}}^{3}$. This lifts to a unitary transformation $\tilde{g}$ in the linear group of isometries. In the projective model of the hyperbolic space, the fixed points of $g$ on $\mathbf{H}_{\mathbb{F}}^{3}$ correspond to (right) eigenvectors of $\tilde{g}$. In the following, we shall forget the 'tilde' from the lift of an isometry and shall use the same symbol for both. When $g$ is an element in $S p(3,1)$, note that the eigenvalues occur in similarity classes. Hence an eigenvalue of $g$ in $S p(3,1)$ will be understood as a similarity class of eigenvalues. We say that two eigenvalues of $g$ are distinct if the corresponding similarity classes of the eigenvalues are disjoint. Two eigenvalues are equal if they belong to the same similarity class. Further note that each similarity class of eigenvalues contains a unique pair of complex conjugate numbers. We further adopt the convention of choosing the eigenvalue $r e^{i \theta}, 0 \leq \theta \leq \pi$ from the similarity class, and we identify the similarity class with this complex eigenvalue. By the Brouwer fixed-point theorem, every isometry of $\mathbf{H}_{\mathbb{F}}^{3}$ has a fixed point in $\mathbf{H}_{\mathbb{F}}^{3} \cup \partial \mathbf{H}_{\mathbb{F}}^{3}$. An isometry $g$ is elliptic if it has a fixed point in $\mathbf{H}_{\mathbb{F}}^{3}$, parabolic if it is non-elliptic and has a unique fixed point on $\partial \mathbf{H}_{\mathbb{F}}^{3}$, and hyperbolic if it is nonelliptic and has exactly two fixed points on $\partial \mathbf{H}_{\mathbb{F}}^{3}$. The conjugacy classification of the isometries follows from [ChGr, section 3]. In particular, every element in $S p(3,1)$ is conjugate to a complex matrix. Using the conjugacy classes, we further refine the above trichotomy of the isometries as follows:

(e) An elliptic element $g$ is regular elliptic if it has mutually distinct eigenvalues; it is compound elliptic if it has two and only two equal eigenvalues. $g$ is called complex elliptic if it has a pair of equal eigenvalues. If $g$ has three equal eigenvalues, it is called screw elliptic. If $g$ has only one similarity class of eigenvalues, it is called simple elliptic. See Table 1 for the classification of elliptic elements. Note that simple elliptics occur only if $\mathbb{F}=\mathbb{H}$. In the complex case, they belong to the center of the group and hence act as the identity.

(h) Suppose $g$ is hyperbolic. It follows from the conjugacy classification that it has a complex eigenvalue outside the unit disc, and one eigenvalue inside the unit disc. The other eigenvalues lie on the unit circle. A hyperbolic isometry $g$ is called a regular hyperbolic if it has a non-real eigenvalue whose norm is different from 1 and the eigenvalues are mutually distinct. $g$ is semiregular if it has a non-real eigenvalue of norm $\neq 1$ and two equal eigenvalues of norm 1. A non-regular hyperbolic isometry has a real eigenvalue different 
TABLE 2. Classification of hyperbolic isometries of $\mathbf{H}_{\mathbb{H}}^{3}$

\begin{tabular}{|l|c|}
\hline Classification & Nature of eigenvalues \\
\hline Regular hyperbolic & $\begin{array}{r}\text { has a non-real eigenvalue of norm } \neq 1 \text { and } \\
\text { eigenvalues are mutually distinct }\end{array}$ \\
\hline $\begin{array}{l}\text { Semi-regular } \\
\text { hyperbolic }\end{array}$ & $\begin{array}{r}\text { has a non-real eigenvalue of norm } \neq 1 \text { and } \\
\text { two equal eigenvalues of norm } 1\end{array}$ \\
\hline Screw hyperbolic & $\begin{array}{r}\text { two and only two real eigenvalues (of norm } \neq 1 \text { ) } \\
\text { Complex if two distinct eigenvalues of norm } 1 \\
\text { Simple if the eigenvalue of norm } 1 \text { has multiplicity two }\end{array}$ \\
\hline Stretch & $\begin{array}{r}\text { all eigenvalues are real numbers } \\
\text { Complex if both } 1 \text { and }-1 \text { are eigenvalues }\end{array}$ \\
& Simple if either 1 or -1 is an eigenvalue of multiplicity two \\
\hline
\end{tabular}

from 1 or -1 . Suppose $g$ is non-regular. If $g$ has two and only two real eigenvalues, it is called screw hyperbolic. A screw hyperbolic element has two eigenvalues on the unit circle. $g$ is a complex screw hyperbolic if it has two distinct eigenvalues on the unit circle; otherwise it is simple screw hyperbolic. $g$ is called a stretch if it has only real eigenvalues: if both 1 and -1 are eigenvalues of a stretch $g$, it is called a complex stretch; if $g$ has either 1 or -1 as an eigenvalue with multiplicity 2, it is called a simple stretch. See Table 2 for the classification of hyperbolic elements. Note that screw hyperbolics occur only if $\mathbb{F}=\mathbb{H}$. When $\mathbb{F}=\mathbb{C}$, the action of the regular hyperbolics and the screw hyperbolics on $\mathbf{H}_{\mathbb{C}}^{3}$ are dynamically the same. It can be seen from the conjugacy classification that a regular hyperbolic can be obtained from a screw hyperbolic by multiplying a central element of the form $\lambda I$, where $|\lambda|=1$, and vice versa. Hence we identify the two classes in $U(3,1)$ and call them loxodromic.

(p) Suppose $g$ is parabolic. If $g$ is unipotent, i.e. all eigenvalues of $g$ are 1 , then it is called a vertical translation or a non-vertical translation according as the minimal polynomial of $g$ is $(x-1)^{2}$ or $(x-1)^{3}$. If $g$ has a nonreal eigenvalue of multiplicity four, then it is called an ellipto-translation or ellipto-parabolic according as the minimal polynomial (of its complex conjugacy class representative) has degree 2 or 3 . When $\mathbb{F}=\mathbb{C}$, these classes do not occur, as their actions on $\mathbf{H}_{\mathbb{C}}^{3}$ are the same as the vertical or non-vertical translations. If $g$ has at least two distinct eigenvalues, it is called a screw translation or a screw parabolic according as the minimal polynomial contains a factor of the form $(x-\lambda)^{2}$ or $(x-\lambda)^{3},|\lambda|=1$. Thus a screw translation has at least two equal eigenvalues. If a screw translation $g$ has three equal eigenvalues, it is called a simple screw translation; it is called a complex screw translation if it has a pair of equal eigenvalues; it is called a compound screw translation if it has two and only two equal eigenvalues. See Table 3 for the classification of parabolic elements.

Given any isometry $g$ of $\mathbf{H}_{\mathbb{F}}^{3}$, it belongs to one of the above classes. 
TABLE 3. Classification of parabolic isometries of $\mathbf{H}_{\mathbb{H}}^{3}$

\begin{tabular}{|l|r|}
\hline Classification & $\begin{array}{c}\text { Nature of eigenvalues } \\
\text { Unipotent }\end{array}$ \\
$\begin{array}{r}\text { all eigenvalues are } 1 \\
\text { Vertical translation if minimal polynomial is }(x-1)^{2} \\
\text { Non-vertical translation if minimal polynomial is }(x-1)^{3}\end{array}$ \\
\hline Ellipto-translation & minimal polynomial is of the form $(x-\lambda)^{2},|\lambda|=1, \lambda \neq \pm 1$ \\
\hline Ellipto-parabolic & minimal polynomial is of the form $(x-\lambda)^{3},|\lambda|=1, \lambda \neq \pm 1$ \\
\hline Screw parabolic & exactly two distinct eigenvalues \\
& minimal polynomial is of the form $(x-\lambda)^{3}(x-\mu)$ \\
\hline Screw translation & at least two distinct eigenvalues \\
& $\begin{array}{r}\text { Sharacteristic polynomial is of the form }(x-\lambda)^{2}(x-\mu)(x-\nu) \\
\text { Complex if } \mu=\nu \text { and } \lambda \neq \mu \\
\text { Compound if } \mu \neq \nu \neq \lambda\end{array}$ \\
\hline
\end{tabular}

We make use of the embedding of $\mathbb{H}$ into $2 \times 2$ complex matrices $M_{2}(\mathbb{C})$. This gives an embedding $A \mapsto A_{\mathbb{C}}$ of $S p(3,1)$ into $G L(8, \mathbb{C})$; cf. (2.1) in section 2, and also see [Go, p. 160], [L, section 2], [Z, section 2]. Using this embedding we obtain the following characterization of the isometries.

Theorem 1.1. Let $A$ be an element in $S p(3,1)$. Let $A_{\mathbb{C}}$ be the corresponding element in $G L(8, \mathbb{C})$.

(A) Let $\beta_{n}=\operatorname{trace}\left(A_{\mathbb{C}}^{n}\right)$. Then $A$ acts as a hyperbolic isometry of $\mathbf{H}_{\mathbb{H}}^{3}$ if and only if $\left\{\beta_{n}\right\}$ is a divergent sequence. For $A$ elliptic or parabolic, $\left\{\beta_{n}\right\}$ is bounded; in fact, for all $n,\left|\beta_{n}\right| \leq 8$.

(B) The characteristic polynomial of $A_{\mathbb{C}}$ is of the form

$$
\chi_{A}(x)=x^{8}-a x^{7}+b x^{6}-c x^{5}+d x^{4}-c x^{3}+b x^{2}-a x+1,
$$

where $a, b, c, d$ are real numbers. Define

$$
\begin{gathered}
H=\frac{1}{6}\left[(b-4)-\frac{3}{8} a^{4}\right], \\
G=\frac{1}{4}\left[(3 a-c)+\frac{1}{2} a(b-4)-\frac{1}{8} a^{3}\right], \\
I=\frac{1}{12}(b-4)^{2}+\frac{1}{4} a(3 a-c)+2(1-b)+d, \\
J=H I-G^{2}-4 H^{3}, \text { and } \Delta=I^{3}-27 J^{2} .
\end{gathered}
$$

Then we have the following:

1. A acts as a regular hyperbolic if and only if $\Delta<0$.

2. $A$ acts as a regular elliptic if and only if $\Delta>0$ and $I<12 H^{2}$.

3. A acts as either complex screw hyperbolic, complex stretch, compound elliptic, or compound screw translation if and only if $\Delta=0, H<0$ and $0<I<12 H^{2}$. Suppose $A$ is not hyperbolic. Then $A$ acts as a compound elliptic, resp. compound 
screw translation, if the degree of the minimal polynomial of $A_{\mathbb{C}}$ is 3 , resp. $4 . A$ acts as semi-regular hyperbolic if and only if $\Delta=0, H<0$ and $I>12 H^{2}$.

4. A acts as either simple screw hyperbolic, simple stretch, complex screw translation, or complex elliptic if and only if $\Delta=0, H<0$ and $I=12 H^{2}$. Suppose $A$ is not hyperbolic. Then $A$ acts as a complex elliptic, resp. a complex screw translation, if the degree of the minimal polynomial of $A_{\mathbb{C}}$ is 2 , resp. 3.

5. Suppose $A$ acts as either screw hyperbolic or stretch. Then $A$ acts as a screw hyperbolic if and only if $4(a+c)^{2} \neq(2 b+d+2)^{2}$. Suppose the equality holds; i.e. $A$ acts as a stretch. Then $A$ acts as a simple or complex stretch according as $a \neq-c$, or $a=-c$.

6. A acts as either screw elliptic, simple screw translation, or screw parabolic if and only if $\Delta=0, H<0$ and $I=0$. Further $A$ acts as screw elliptic, resp. simple screw translation, resp. screw parabolic, if the degree of the minimal polynomial of $A_{\mathbb{C}}$ is 2 , resp. 3, resp. 4.

7. A acts as either simple elliptic, unipotent, ellipto-translation, or elliptoparabolic if and only if $\Delta=0, H=0$ and $I=0$. In these cases, $|a| \leq 8,|b| \leq 28$, $|c| \leq 56$ and $|d| \leq 70$. The equality holds if and only if $A \neq \pm I$ and $A$ is unipotent.

Suppose $A$ is non-unipotent. Then $A$ acts as a simple elliptic, resp. elliptotranslation, resp. ellipto-parabolic, if the degree of the minimal polynomial of $A_{\mathbb{C}}$ is 1, resp. 2, resp. 3 .

8. Suppose $A$ is unipotent. Then $A$ acts as a vertical translation, resp. nonvertical translation, if the degree of the minimal polynomial of $A_{\mathbb{C}}$ is 2 , resp. 3 .

Note that the well-known Newton's formulas (see [R, Theorem 1.3.19], $\mathrm{Mc}$ ) express the numbers $\beta_{n}$ in terms of the coefficients of the characteristic polynomials of $A_{\mathbb{C}}$. The approach used above also restricts to $U(3,1)$. In this case, the above embedding restricts to the embedding $A \rightarrow A_{\mathbb{R}}$ of $U(3,1)$ into $G L(8 ; \mathbb{R})$. This provides the following characterization of the isometries of $\mathbf{H}_{\mathbb{C}}^{3}$.

Corollary 1.2. Let $A$ be an element in $U(3,1)$. Let $A_{\mathbb{R}}$ be the corresponding element in $G L(8, \mathbb{R})$.

(A) Let $\alpha_{n}=\operatorname{trace}\left(A^{n}\right)$. Then $A$ acts as a hyperbolic isometry of $\mathbf{H}_{\mathbb{C}}^{3}$ if and only if $\left\{\alpha_{n}\right\}$ is a divergent sequence. For $A$ elliptic or parabolic, $\left\{\alpha_{n}\right\}$ is bounded; in fact, for all $n,\left|\alpha_{n}\right| \leq 4$.

(B) The characteristic polynomial of $A_{\mathbb{R}}$ is of the form

$$
\chi_{A}(x)=x^{8}-a x^{7}+b x^{6}-c x^{5}+d x^{4}-c x^{3}+b x^{2}-a x+1,
$$

where $a, b, c, d$ are real numbers. Let $G, H, I, J$ and $\Delta$ be as in the above theorem. Then we have the following:

1. A acts as a regular elliptic if and only if $\Delta>0$ and $I<12 H^{2}$.

2. A acts as a loxodromic if and only if one of the following conditions holds.

(i) $\Delta<0$.

(ii) $A$ is hyperbolic, $\Delta=0, H<0$, and either $I>12 H^{2}$, $\operatorname{trace}(A) \neq r \lambda$ for $r>0,|\lambda|=1$, or $0<I \leq 12 H^{2}, 4(a+c)^{2} \neq(2 b+d+2)^{2}$.

3. $A$ acts as a stretch if and only if $A$ is hyperbolic, and $\Delta<0, H<0,0<I \leq$ $12 H^{2}, 4(a+c)^{2}=(2 b+d+2)^{2}$. Further $A$ is simple or complex according as $a \neq-c$ or $a=-c$. Also, if $\Delta=0, H<0, I>12 H^{2}$ and $\operatorname{trace}(A)=r \lambda, r>0,|\lambda|=1$, then $A$ acts as simple stretch.

4. A acts as either compound elliptic or compound screw translation if and only if $A$ is not hyperbolic and $\Delta=0, H<0$ and $0<I<12 H^{2}$. Further, $A$ acts as a 
compound elliptic, resp. a compound screw translation, if the degree of the minimal polynomial of $A_{\mathbb{C}}$ is 3 , resp. 4 .

5. A acts as either a complex screw translation or complex elliptic if and only if $A$ is not hyperbolic and $\Delta=0, H<0$ and $I=12 H^{2}$. Further, $A$ acts as a complex elliptic, resp. complex screw translation, if the degree of the minimal polynomial of $A_{\mathbb{C}}$ is 2 , resp. 3.

6. A acts as either screw elliptic, simple screw translation, or screw parabolic if and only if $\Delta=0, H<0$ and $I=0$. Further $A$ acts as a screw elliptic, resp. simple screw translation, resp. screw parabolic, if the degree of the minimal polynomial of $A_{\mathbb{C}}$ is 2 , resp. 3, resp. 4.

7. A acts as a unipotent element if and only if $A \neq \lambda I,|\lambda|=1$ and $\Delta=0$, $H=0$ and $I=0$. Further, $A$ acts as a vertical translation, resp. non-vertical translation, if the degree of the minimal polynomial of $A_{\mathbb{C}}$ is 2 , resp. 3 .

8. $A$ acts as the identity if and only if $A=\lambda I,|\lambda|=1$.

\section{A simple characterization of the isometries of $\mathbf{H}_{\mathbb{F}}^{n}$}

For the definition and basic properties of the complex and quaternionic hyperbolic spaces, see [ChGr, $\left[\mathrm{Mo}\right.$. The conjugacy classification of the isometries of $\mathbf{H}_{\mathbb{F}}^{\mathbf{n}}$ can be obtained in $\mathrm{ChGr}$, section 3].

Lemma 2.1. Let $A$ be an element in $U(n, 1)$. For $n \geq 1$, let $\alpha_{n}=$ trace $A^{n}$.

(i) $A$ acts as a hyperbolic isometry of $\mathbf{H}_{\mathbb{C}}^{\mathbf{n}}$ if and only if there exists a positive integer $m$ such that $\left|\alpha_{m}\right|>n+1$. Alternatively, $A$ acts as a hyperbolic isometry if and only if $\left\{\alpha_{n}\right\}$ is a divergent sequence.

(ii) $A$ acts as an elliptic or parabolic element if and only if for all $n,\left|\alpha_{n}\right| \leq n+1$. Further, $A$ acts as a unipotent element if and only if $\left|\alpha_{n}\right|=n+1$.

Proof. Let $A$ act as a hyperbolic isometry. Hence $A$ must have eigenvalues $\lambda, \lambda^{-1}$ such that $|\lambda|>1$. The other eigenvalues, say $u_{1}, \ldots, u_{n-1}$, belong to the unit circle; i.e. for each $i,\left|u_{i}\right|=1$. Hence

$$
\begin{gathered}
\operatorname{trace} T=\lambda+\lambda^{-1}+\sum_{i=1}^{n-1} u_{i}, \\
\operatorname{trace} T^{n}=\lambda^{n}+\lambda^{-n}+\sum_{i=1}^{n-1} u_{i}^{n} .
\end{gathered}
$$

Since $|\lambda|=1$ and $\left|u_{i}\right|=1$, we have

$$
\left|\operatorname{trace} T^{n}\right| \geq|\lambda|^{n}-n \geq r^{n}-n,
$$

where $r \in \mathbb{R}$ is such that $|\lambda| \geq r>1$. Since $r>1$, by the Archimedean property of the real numbers, one can choose $m$ such that $r^{n}>2 n+1$; hence $\left|\alpha_{m}\right|>n+1$.

For $A$ elliptic or parabolic, all the eigenvalues lie on the unit circle $\mathbb{S}^{1}$; hence

$$
\left|\operatorname{trace} T^{n}\right|=\left|\sum_{i=1}^{n+1} u_{i}\right| \leq n+1 .
$$

Thus the sequence $\left\{\alpha_{n}\right\}$ is bounded.

Note that a unipotent element has only 1 as an eigenvalue. For each $n, \alpha_{n}$ is the sum of $n$-th powers of eigenvalues of $A$. Hence $\left|\alpha_{n}\right|=n+1$ for all $n$ if and only if $A$ acts as a unipotent element. 
Let $A \in S p(n, 1)$. Write $\mathbb{H}=\mathbb{C} \oplus \mathbf{j} \mathbb{C}$. Express $A=A_{1}+\mathbf{j} A_{2}$, where $A_{1}, A_{2} \in$ $M_{2(n+1)}(\mathbb{C})$. This gives an embedding $A \mapsto A_{\mathbb{C}}$ of $S p(n, 1)$ into $G L(2(n+1), \mathbb{C})$, where

$$
A_{\mathbb{C}}=\left(\begin{array}{cc}
A_{1} & -\overline{A_{2}} \\
A_{2} & \overline{A_{1}}
\end{array}\right) .
$$

Lemma 2.2. Let $A$ be an element in $S p(n, 1)$. Let $A_{\mathbb{C}}$ be the corresponding element in $G L(2(n+1), \mathbb{C})$. For $n \geq 1$, let $\beta_{n}=$ trace $A_{\mathbb{C}}^{n}$.

(i) A acts as a hyperbolic isometry if and only if there exists a positive integer $m$ such that $\left|\beta_{m}\right|>2(n+1)$. Alternatively, $A$ acts as a hyperbolic isometry if and only if $\left\{\beta_{n}\right\}$ is a divergent sequence.

(ii) $A$ acts as an elliptic or parabolic element if and only if for all $n,\left|\beta_{n}\right| \leq$ $2(n+1)$. Further, $A$ is unipotent if and only if $\left|\beta_{n}\right|=2(n+1)$.

Proof. Suppose $A$ is hyperbolic. Then $A$, consequently $A_{\mathbb{C}}$, must have eigenvalues $\lambda, \lambda^{-1}$ such that $|\lambda|>1$. For $A$ elliptic or parabolic, all the eigenvalues of $A_{\mathbb{C}}$ are unit complex numbers. Now, the proof is completely analogous to the proof of Lemma 2.1.

Remark 2.3. In the above lemmas, it is, in fact, possible to determine the numbers $\alpha_{n}$ and $\beta_{n}$ from the coefficients of the characteristic polynomials of $A$ and $A_{\mathbb{C}}$ respectively. This is possible by virtue of the well-known Newton's identities; cf. [R, Theorem 1.3.19], [Mc].

\section{Proof of Theorem 1.1}

In order to prove Theorem 1.1, we shall heavily use the well-known criterion of the nature of roots of a quartic or a bi-quadratic equation. We follow the criterion in [Re; also see [D, Chapter IV], $\mathrm{BP}$, Chapter VI].

Let $A$ be an element in $S p(3,1)$. Note that the characteristic polynomial $\chi_{A_{\mathbb{C}}}(x)$ of $A_{\mathbb{C}}$ is an invariant of the conjugacy class of $A$. It follows from the conjugacy class representatives in $S p(3,1)$ that $\chi_{A_{\mathbb{C}}}(x)$ is self-dual; i.e. if $\lambda \in \mathbb{C}$ is a root of $\chi_{A_{\mathbb{C}}}(x)$, so is $\lambda^{-1}$. Further, if $\lambda$ is an eigenvalue, then so is $\bar{\lambda}^{-1}$; cf. Gol, Lemma 6.2.5, p. 205]. It follows that if $\lambda$ is a root of the characteristic polynomial, so is $\bar{\lambda}$. Hence the characteristic polynomial of $A_{\mathbb{C}}$ is of the form

$$
\chi_{A_{\mathbb{C}}}(x)=x^{8}-a x^{7}+b x^{6}-c x^{5}+d x^{4}-c x^{3}+b x^{2}-a x+1,
$$

where $a, b, c, d \in \mathbb{R}$. Write $\chi_{A_{\mathbb{C}}}(x)=x^{4} g(x)$, where

$$
g(x)=\left(x^{4}+x^{-4}\right)-a\left(x^{3}+x^{-3}\right)+b\left(x^{2}+x^{-2}\right)-c\left(x+x^{-1}\right)+d .
$$

Expanding the terms in the brackets, we have

$g(x)=\left(x+x^{-1}\right)^{4}-a\left(x+x^{-1}\right)^{3}+(b-4)\left(x+x^{-1}\right)^{2}-(c-3 a)\left(x+x^{-1}\right)+(d-2 b+2)$.

Thus $g(x)$ can be expressed as a polynomial in $\left(x+x^{-1}\right)$, and we consider $g_{A}$ in terms of the variable $t=\left(x+x^{-1}\right)$,

$$
g_{A}(t)=t^{4}-a t^{3}+(b-4) t^{2}-(c-3 a) t+(d-2 b+2) .
$$

Since $\chi_{A_{\mathbb{C}}}(x)$ is a conjugacy invariant, so is $g_{A}(t)$. If $\alpha$ is a root of $\chi_{A_{\mathbb{C}}}(x)$, then $\alpha+\alpha^{-1}$ is a root of $g_{A}(t)$. Hence the nature of the roots of $g_{A}(t)$ is determined by the nature of the roots of $\chi_{A_{\mathbb{C}}}(x)$. 
We write $g_{A}(t)$ in the standard form as

$$
g_{A}(t)=t^{4}+4 B t^{3}+6 C t^{2}+4 D t+E,
$$

where $B=-\frac{1}{4} a, C=\frac{1}{6}(b-4), D=\frac{1}{4}(3 a-c), E=d-2 b+2$. Set $H=C-B^{2}$, $G=D-3 B C+2 B^{3}, I=E-4 B D+3 C^{2}$. In terms of $a, b, c, d$,

$$
\begin{array}{r}
H=\frac{1}{6}\left[(b-4)-\frac{3}{8} a^{4}\right], \\
G=\frac{1}{4}\left[(3 a-c)+\frac{1}{2} a(b-4)-\frac{1}{8} a^{3}\right], \\
I=\frac{1}{12}(b-4)^{2}+\frac{1}{4} a(3 a-c)+2(1-b)+d .
\end{array}
$$

Setting $Z=A t+B$, one may further reduce the equation $g_{A}(t)=0$ to the form (see [BP, p. 116])

$$
Z^{4}+Q Z^{2}+R Z+S=0
$$

where

$$
Q=6 H, R=4 G, S=I-\frac{1}{12} Q^{2} .
$$

The discriminant of the equation $g_{A}(t)=0$ is given by

$$
\Delta=I^{3}-27 J^{2}
$$

where $J=H I-G^{2}-4 H^{3}$. The nature of the roots of $g_{A}(t)=0$ can be completely determined in terms of $H, G, I$ and the discriminant $\Delta$; see [Re, $\mathrm{BP}$.

Suppose $A$ acts as a hyperbolic isometry. Suppose the roots of $\chi_{A_{\mathbb{C}}}(x)$ are $r e^{i \beta}$, $r e^{-i \beta}, r^{-1} e^{i \beta}, r^{-1} e^{-i \beta}, e^{i \theta}, e^{-i \theta}, e^{i \phi}$ and $e^{-i \phi}$. The roots of $g_{A}(t)$ are given by

$$
t_{1}=r e^{i \beta}+r^{-1} e^{i \beta}, t_{2}=r e^{-i \beta}+r^{-1} e^{i \beta}, t_{3}=2 \cos \theta, t_{4}=2 \cos \phi .
$$

(1) Thus $g_{A}(t)$ has two distinct real and two distinct non-real roots if and only if $\Delta<0$. From the expression of the roots it is clear that this is precisely the case when $\beta \neq 0$; i.e. $A$ is regular hyperbolic.

If $A$ is semi-regular, then $\theta=\phi$ and it follows from [Re that $H<0, I>12 H^{2}$.

(2) Suppose $\beta=0$ or $\pi$; i.e. $A$ acts as a screw hyperbolic or a stretch. Thus $g_{A}(t)$ has at least two real and equal roots $r+r^{-1}$. For $r>0$, note that $r+r^{-1}>2$.

First suppose that $A$ acts as a stretch. Then there are two cases: either $A$ is simple or $A$ is complex, i.e. either $g_{A}(t)$ has a root 2 , or -2 , of multiplicity two or $g_{A}(t)$ has both 2 and -2 as roots. Suppose both $g_{A}(2)=0$ and $g_{A}(-2)=0$. This implies that

$$
2 b+d+2=2(a+c), 2 b+d+2=-2(a+c),
$$

which is possible if and only if $a=-c$. Thus if $a \neq-c$ and $(2 b+d+2)^{2}=4(a+c)^{2}$, then either $g_{A}(2)=0$ or $g_{A}(-2)=0$, but not both, and hence $A$ acts as a simple stretch. If $g_{A}(t)$ has at least one root in the open interval $(-2,2)$, then $A$ acts as a screw hyperbolic.

(i) Suppose $A$ acts as a complex screw hyperbolic or a complex stretch, i.e. $\theta \neq \phi$. In this case $g_{A}(t)$ has two and only two equal roots. Hence, $\Delta=0$, $H<0$, and $-3 H^{2}<S<9 H^{2}$ i.e. $0<I<12 H^{2}$. Further, if $A$ acts as a complex stretch, then it follows from the above that $a=-c$ and $(2 b+d+2)^{2}=4(a+c)^{2}=0$. 
(ii) Suppose $A$ acts as a simple screw hyperbolic or a simple stretch, i.e. $\theta=\phi$. Since $g_{A}(t)$ has two pairs of equal real roots, $\Delta=0, H<0$, and $S=9 H^{2}$, i.e. $I=12 H^{2}$. Further, if $A$ acts as a simple stretch, then $a \neq-c$ and $(2 b+d+2)^{2}=4(a+c)^{2}$.

Suppose $A$ acts as an elliptic or a parabolic isometry. Suppose the roots of $\chi_{A_{\mathbb{C}}}(x)$ are $e^{i \theta_{k}}, e^{-i \theta_{k}}, k=1,2,3,4$.

(3) Suppose $A$ acts as a regular elliptic; i.e. for all $i, j, \theta_{i} \neq \theta_{j}$. Thus $g_{A}(t)$ has four real and mutually distinct roots. Hence, $\Delta>0$ and $I<12 H^{2}$.

(4) Suppose $A$ acts as a compound elliptic or a compound screw translation; i.e. exactly two of the $\theta_{j}$ 's are equal. Thus $g_{A}(t)$ has two and only two equal roots. Hence $\Delta=0, H<0$ and $0<I<12 H^{2}$. Further if $A$ is a compound elliptic, $A_{\mathbb{C}}$ has a minimal polynomial of the form $(x-\lambda)(x-\mu)(x-\delta)$. If $A$ is a compound screw translation, the minimal polynomial is of the form $(x-\lambda)^{2}(x-\mu)(x-\delta)$.

(5) Suppose $A$ acts as a screw elliptic, or a simple screw translation, or a screw parabolic. Then it has three equal eigenvalues, and hence $g_{A}(t)$ has three equal roots; hence $\Delta=0, H<0$ and $I=0$. If $A$ acts as a simple screw translation, then $A_{\mathbb{C}}$ has minimal polynomial $m_{A_{\mathbb{C}}}(x)$ of the form $(x-\lambda)^{2}(x-\mu), \lambda, \mu \in \mathbb{S}^{1}$. If $A$ acts as a screw parabolic, $m_{A_{\mathbb{C}}}(x)$ is of the form $(x-\lambda)^{3}(x-\mu), \lambda, \mu \in \mathbb{S}^{1}$. For $A$ a screw elliptic, $m_{A_{\mathbb{C}}}(x)$ is of the form $(x-\lambda)(x-\mu)$. Thus the degree of the minimal polynomial distinguishes the three classes.

(6) Suppose $A$ acts as a simple elliptic, or a unipotent, or an ellipto-translation, or an ellipto-parabolic. Then $g_{A}(t)$ has four equal roots. Consequently, we have $\Delta=0$, $H=0$ and $I=0$. Next the degree of $m_{A_{\mathbb{C}}}(x)$ distinguishes a vertical translation or ellipto-translation from a non-vertical translation or ellipto-parabolic. Further, $A$ is a vertical or non-vertical translation if and only if 1 is the only eigenvalue. Hence we must have $|a|=8,|b|=28,|c|=56,|d|=70$. For $A$ non-unipotent, $|a|<8$, $|b|<28,|c|<56,|d|<70$.

(7) Suppose $A$ acts as a complex screw translation or a complex elliptic. Then $g_{A}(t)$ has a pair of equal roots; hence $\Delta=0, H<0$, and $I=12 H^{2}$. Further the degree of $m_{A_{\mathbb{C}}}(x)$ distinguishes these classes.

Finally, Lemma 2.2 distinguishes a hyperbolic isometry from a parabolic or an elliptic isometry.

This completes the proof of the theorem.

\section{Summary}

For the reader's convenience, we summarize the algorithm to characterize isometries of $\mathbf{H}_{\mathbb{H}}^{3}$. The complex hyperbolic case is similar.

Let $A \in S p(3,1)$ be given.

1. First use Table 4. If $\Delta>0$ or $\Delta<0$, we are done. Otherwise proceed to the next step.

2. Suppose $\Delta=0$. Compute $H$.

Case A. Suppose $H=0$. If $(A-I)^{4}=0$, i.e. all the eigenvalues are 1 , then $A$ is unipotent. It is vertical or non-vertical according as the minimal polynomial is $(x-1)^{2}$ or $(x-1)^{3}$.

Suppose $A$ is not unipotent. Then it is simple elliptic or parabolic according as the minimal polynomial of $A_{\mathbb{C}}$ is linear or non-linear. If $A$ is parabolic, then it is ellipto-translation or ellipto-parabolic according as the degree of the minimal polynomial is 2 or 3 . 
TABLE 4. $\Delta$-classification of isometries of $\mathbf{H}_{\mathbb{H}}^{3}$

\begin{tabular}{|l|c|}
\hline$\Delta$ & Type of isometry \\
\hline$<0$ & Regular hyperbolic \\
\hline$>0$ & Regular elliptic \\
\hline$=0$ & Parabolic, non-regular hyperbolic or non-regular elliptic \\
\hline
\end{tabular}

Case B. Suppose $H \neq 0$. Then we must have $H<0$. Compute $I$.

(a) Suppose $I=0$. If $A_{\mathbb{C}}$ has a minimal polynomial of degree 2 , then $A$ is screw elliptic. It is a simple screw translation, resp. screw parabolic, if the degree of the minimal polynomial is 3 , resp. 4 .

(b) Suppose $I \neq 0$. Compute $I-12 H^{2}$.

(b (i)) Suppose $I-12 H^{2}>0$. Then $A$ is semi-regular hyperbolic. Suppose $I-12 H^{2}<0$. If $\left\{\beta_{n}\right\}$ is divergent, then $A$ is complex screw hyperbolic or complex stretch. Otherwise, it is a compound elliptic, resp. compound screw translation, if the degree of the minimal polynomial is 3 , resp. 4 .

(b (ii)) Suppose $I-12 H^{2}=0$. If $\left\{\beta_{n}\right\}$ is divergent, then $A$ is simple screw hyperbolic or simple stretch. Suppose $A$ is non-hyperbolic. Then it is a complex elliptic, resp. complex screw translation, if the degree of the minimal polynomial of $A_{\mathbb{C}}$ is 2 , resp. 3 .

Suppose $A$ is a screw hyperbolic or a stretch in the above two cases. Then $A$ is a screw hyperbolic if and only if $\chi_{A_{\mathbb{C}}}(1) \neq 0$ and $\chi_{A_{\mathbb{C}}}(-1) \neq 0$. If $A$ is a stretch, then it is simple if either $\chi_{A_{\mathbb{C}}}(1)=0$ or $\chi_{A_{\mathbb{C}}}(-1)=0$, but not both; it is complex if $\chi_{A_{\mathbb{C}}}(1)=0$ and $\chi_{A_{\mathbb{C}}}(-1)=0$.

\section{ACKNOWLEDGEMEnTS}

The author thanks John Parker and Jose Seade for their comments on a first draft of this article.

\section{REFERENCES}

[Be] A. F. Beardon, The geometry of discrete groups, Berlin, New York: Springer-Verlag, 1983. MR698777 (85d:22026)

[BP] W. S. Burnside and A. W. Patron, The theory of equations with an introduction to the theory of binary algebraic forms, Dublin University Press Series, 1881.

[CaGo] W. S. Cao and K. Gongopadhyay, Algebraic characterization of isometries of the complex and the quaternionic hyperbolic planes. Geom. Dedicata 157, no. 1 (2012), 23-39. MR2893478

[D] L. E. Dickson, Elementary Theory of Equations. John Wiley \& Sons, New York, 1914.

[ChGr] S. S. Chen and L. Greenberg, Hyperbolic spaces, Contributions to analysis. Academic Press, New York, 1974, 49-87. MR0377765 (51:13934)

[Gol] W. M. Goldman, Complex Hyperbolic Geometry. Oxford University Press, 1999. MR1695450 (2000g:32029)

[Go] K. Gongopadhyay, Algebraic characterization of the isometries of the hyperbolic 5-space. Geom. Dedicata (1) 144 (2010), 157-170. MR2580424 (2011a:57004)

[L] H. C. Lee, Eigenvalues and canonical forms of matrices with quaternion coefficients. Proc. Roy. Irish Acad. Sect. A. 52 (1949), 253-260. MR0036738 (12:153i)

[Mc] I. G. Macdonald, Symmetric functions and Hall polynomials. Oxford University Press, 1995. MR.1354144 (96h:05207) 
[Mo] G.D. Mostow, Strong Rigidity of Locally Symmetric Spaces. Annals of Math. Studies, 78, Princeton University Press; University of Tokyo Press, 1973. MR0385004 (52:5874)

[Na] J.-P. Navarrete. The trace function and complex Kleinian groups in $\mathbb{P}_{\mathbb{C}}^{2}$, Internat. J. Math. 19, no. 7 (2008), 865-890. MR2437075 (2009g:32056)

[Re] E. L. Rees, Graphical discussion of the roots of a quartic equation, Amer. Math. Monthly, 29, no. 2 (1922), pp. 51-55. MR.1519936

[R] L. H. Rowen, Polynomial Identities in Ring Theory, Pure and Applied Mathematics, Academic Press (London), 1980. MR576061(82a:16021)

[SV] J. Seade and A. Verjovsky. Actions of discrete groups on complex projective spaces. Contemporary Math., 269, Amer. Math. Soc., 2001, 155-178. MR.1810539 (2002d:32024)

[Z] F. Zhang, Quaternions and matrices of quaternions. Linear Algebra Appl. 251 (1997), 21-57. MR 1421264 (97h:15020)

Indian Institute of Science Education and Research (IISER) Mohali, Knowledge City, S.A.S. Nagar, Sector 81, Mohali 140306, India

E-mail address: krishnendug@gmail.com 